Referencia para citar este artículo: Acosta-Silva, D. A. (2017). Tras las competencias de los nativos digitales: avances de una metasíntesis. Revista Latinoamericana de Ciencias Sociales, Niñez y Juventud, 15(1), pp. 471-489.

\title{
Tras las competencias de los nativos digitales: avances de una metasíntesis*
}

\author{
DaVID Arturo A Costa-SILVA** \\ Investigador Corporación Universitaria Unitec, Colombia.
}

\section{Artículo recibido en junio 14 de 2016; artículo aceptado en agosto 8 de 2016 (Eds.)}

- Resumen (descriptivo): En el presente documento reporto los avances de una metasíntesis realizada sobre investigaciones que en los últimos 15 años han buscado evaluar las competencias digitales de los jóvenes; ello con el fin de establecer si sus resultados soportan las posturas que afirman que los jóvenes y las jóvenes tienen unas competencias digitales generalizadas y de alto nivel. Para tal fin realicé una selección en la literatura de reportes de investigaciones cuyos objetivos se dirigieran al análisis de las competencias digitales, posteriores al planteamiento original de los nativos digitales y que presentaran resultados empíricos. De los artículos obtenidos analicé sus concepciones, métodos y resultados. El producto de este proceso indica que la mayoría de tales estudios concluyen que las aseveraciones sobre las superiores competencias digitales de los sujetos jóvenes no están soportadas empíricamente.

Palabras clave: nativos digitales, competencias digitales, jóvenes, tecnología digital, TIC (Tesauro de Ciencias Sociales de la Unesco).

\section{In search of the competencies of digital natives: progress of a meta-synthesis}

- Abstract (descriptive): This paper reports the first results of a meta-synthesis of research studies over the last 15 years that have sought to evaluate the digital competencies of young people; our purpose was to establish whether these results support the claim that young people have widespread and high-level digital competencies. For this purpose, a screening was performed as part of the literature review, searching for papers that had the objective of analyzing digital competencies following an exploration of the concept of digital natives and the sharing of empirical results. An analysis was conducted for the concepts, methods and results from these studies. The result of this process showed that most of these studies conclude that such claims about young people's digital competencies are not empirically supported.

Key words: digital natives, digital competences, youth, digital technology, ICT (Unesco Social Sciences Thesaurus).

\section{Em busca de competências dos nativos digitais: avanços de uma meta-síntese.}

- Resumo (descritivo): O presente documento reporta os avanços de uma meta-síntese realizada sobre investigações que nos últimos 15 anos buscaram avaliar as competências digitais de

\footnotetext{
Este artículo corto presenta los primeros avances de la investigación «Meta-síntesis (meta-análisis cualitativo) sobre las concepciones y modelos de evaluación de las competencias digitales empleados en estudios realizados en el período 2001-2016». El proyecto ha sido financiado por el Sistema Institucional de Investigación de la Corporación Universitaria Unitec para su realización durante el año 2016 (cód. I-0316013). Se llevó a cabo entre los meses de febrero y noviembre de 2016. Área de conocimiento: Ciencias Sociales, Subárea: Temas especiales.

** Filósofo de la Universidad Nacional de Colombia. Magíster en Educación de la Universidad de La Salle. Candidato a Doctor, Doctorado en Ciencias Sociales, Niñez y Juventud del Centro de Estudios Avanzados en Niñez y Juventud del Cinde y la Universidad de Manizales. Correo electrónico: davidacostasilva@gmail.com
} 
jovens; isso com o fim de estabelecer se seus resultados suportam as posturas que afirmam que os jovens possuem algunas competências digitais generalizadas e de alto nível. Para tanto realizou-se uma seleção de literatura de relatórios de investigação cujo os objetivos se dirigiram a análise das competências digitais que, foram posteriores ao planteamento original dos nativos digitais e que apresentaram resultados empíricos. Dos artigos obtidos analiszram-se seus concepções, métodos e resultados. O produto deste processo nos diz que a maioria de tais estudos conncluem que as declarações sobre as superiores competências digitais dos jovens não possuem suporte empírico.

Palavras-chave: nativos digitais, competências digitais, jovens, tecnologia digital, TIC (Thesaurus de Ciências Sociais da Unesco).

-1. Introducción. -2. Método. -2.1. Descripción del estudio. -2.2. Estrategias de análisis. -3. Resultados y discusión. -3.1. Concepciones y teorizaciones sobre la competencia digital. -3.2. Métodos e instrumentos. -3.3. Análisis de los resultados. -4. Conclusiones. -Lista de Referencias.

\section{Introducción}

Ya son 15 años desde que Prensky (2001) propuso la irrupción de una nueva generación a la que calificó como fundamentalmente diferente a las anteriores, gracias a ser contemporánea de uno de los acontecimientos capitales de los últimos 50 años: la popularización de la tecnología digital a comienzos de la década de los 80 y su masificación -al menos en los niveles socioeconómicos más altos- en los 90 asociada a la aparición de Internet en los hogares. De esta manera, el hecho de ser la primera generación en crecer rodeada de la tecnología digital significaría, según la lectura de Prensky, que mantendría una relación con aquella caracterizada especialmente por su «naturalidad»; y precisamente para subrayar lo anterior, llamó a sus miembros nativos digitales, los hablantes de la lengua digital. El resto de la población, excluido de esta supuesta estrecha relación, serían entonces apenas inmigrantes digitales, relegados a mantenerse siempre «con un pie en el pasado» (p. 2). Esta cercanía a lo digital les daría a los nativos una serie de características pronunciadas y definitorias; entre otras, preferirían la información gráfica a la textual, prosperarían gracias a las gratificaciones inmediatas, así como también pensarían y procesarían información de forma diferente a cualquier «inmigrante».

La potencia de esta categoría es indudable: encapsulaba tres fenómenos que calaban cada vez con mayor fuerza en el imaginario colectivo de occidente, generando escozor $y$,

472 en muchos, abiertamente miedo. En primer lugar, la extrema rapidez con la que lo digital se colaba en nuestra cotidianeidad; segundo, la facilidad con la que aparentemente los jóvenes la manejaban, así como el papel preponderante de aquella en sus vidas; $y$, tercero, el innegable impacto de lo anterior en los procesos educativos. No obstante, la fragmentación de la sociedad en nativos e inmigrantes sirvió para señalar otro capítulo en el proverbial pánico social frente a la tecnología, que sería luego, como podría esperarse, avivado, hiperbolizado y dramatizado por los medios de comunicación $\mathrm{y}$ por ciertos autores insidiosos.

La notoriedad adquirida por la etiqueta de los nativos digitales finalmente llamaría la atención de la academia más o menos un lustro después de la propuesta de Prensky. Varios investigadores e investigadoras comenzaron a preguntarse por sus sustentos fácticos -e incluso teóricos-, y los resultados que obtuvieron empezaron a resquebrajar dicha construcción: en primer lugar, se ha mostrado que el acceso a la tecnología varía ampliamente entre las personas jóvenes (Bennett, Maton \& Kervin, 2008, Thinyane, 2010), así que, de entrada, es imposible calificar a todos los jóvenes y las jóvenes como nativos digitales cuando millones de ellos y ellas ni siquiera tienen acceso a ésta; asimismo, también se ha sugerido que en esta generación hay personas que rara vez emplean la tecnología (Bennett, Maton \& Kervin, 2008, Helsper \& Eynon, 2010), y quienes sí lo hacen, difieren ampliamente en las características de dicho uso, así como en su nivel de desempeño 
(Hargittai \& Hinnant, 2008, Kennedy, Judd, Churchward, Gray \& Krause, 2008, Margaryan, Littlejohn \& Vojt, 2011), lo que implica una fuerte heterogeneidad contraria a la imagen propuesta por la etiqueta; por otra parte, varios estudios han llegado a la conclusión de que no existen diferencias significativas en el uso de la tecnología por las diferentes generaciones (Bennett, Maton \& Kervin, 2008, Bullen, Morgan, Belfer \& Qayyum, 2009, Dias da Silva, da Silva \& do Amaral, 2014, Fajardo, Villalta \& Salmerón, 2015, Helsper \& Eynon, 2010).

Finalmente, un grupo importante de estudios ha buscado analizar el foco de la postura de Prensky y de otros que han descrito a los nativos digitales (Oblinger \& Oblinger, 2005, Howe \& Strauss, 2000, Tapscott, 1998): que la gran brecha entre éstos y los inmigrantes radica en que los primeros tienen unas habilidades y conocimientos más sofisticados y cuantitativamente superiores a los segundos, lo que les permite navegar lo digital con total tranquilidad. No obstante, el problema es que al traducir esta postura al lenguaje académico, las interpretaciones se han multiplicado; así, dichas habilidades han sido entendidas como alfabetismo digital (digital literacy, Gilster, 1997, Eshet-Alkali \& Amichai-Hamburger, 2004), habilidades en Internet (Internet skills, Van Deursen, Van Dijk \& Peters, 2011), habilidades digitales (digital skills, Gui \& Argentin, 2011), alfabetismo en Internet (Internet literacy, Livingstone, Bober \& Helsper, 2005) y, desde luego, como competencias digitales.

No obstante, la dificultad que presentan estas propuestas no es simplemente una cuestión de denominaciones, sino más bien de solapamientos. Si bienmuchas de ellas provienen de agendas de investigación diferentes, sus objetivos no permiten trazar entre ellas límites precisos. Y a esta situación se le suma el hecho de que hay además diferentes interpretaciones en cada una de estas posturas, lo que las acerca o las aleja de las demás -dependiendo del énfasis que le dé cada académico-, sin que una concepción siquiera compartida por una mayoría sea posible al día de hoy. Por ejemplo, el alfabetismo digital usualmente ha sido interpretado exclusivamente como el manejo de la información digital; no obstante, no es difícil encontrar interpretaciones que lo consideren como sinónimo del constructo de las competencias digitales (Li \& Ranieri, 2010).

Ahora bien, respecto a la elección de éste y otros trabajos de la expresión 'competencias', las críticas que esta opción puede recibir ya son añejas (Brunet \& Belzunegui, 2003). Pero sin entrar en ese espinoso terreno -para el cual no hay espacio en este texto-, no podemos olvidar que dicha elección puede ser una cuestión de contexto. Por ejemplo, su aparición en la gran mayoría de los artículos que analizaré a continuación se explica por la inserción de éstos en el marco europeo de educación, en donde ésta es la terminología adaptada (Comisión Europea, 2007), así como también lo es en algunos países latinoamericanos. Por su parte, en mi caso particular, dicha elección tiene que ver con una apuesta teórica que incluye las competencias como una de las etapas del desarrollo de las potencialidades humanas, que parte de las capacidades básicas hasta llegar al virtuosismo (Acosta-Silva \& Vasco, 2013).

Pero volviendo a los estudios, la pregunta que nos podemos hacer es, después de todos estos años, ¿las investigaciones realizadas en este campo han socavado definitivamente las bases de esta famosa etiqueta, en especial en lo referido al supuesto dominio de la tecnología por los jóvenes? En este marco, en el proyecto de investigación -del cual en estas líneas presento sus primeros avances- he pretendido realizar una metasíntesis de la literatura científica desde la publicación de Prensky, buscando determinar cómo han sido entendidas las competencias digitales y sus constructos paralelos, de qué manera han sido evaluadas en los jóvenes y qué resultados se ha obtenido. No obstante, mi objetivo en lo que sigue no será presentar los resultados de toda la investigación, sino que me centraré en el análisis de un subgrupo de dichas investigaciones: aquellas que directamente han buscado analizar las competencias digitales. A partir de sus resultados, y de manera especial, me interesa responder la cuestión sobre si 
todavía se puede seguir manteniendo que los jóvenes y las jóvenes tienen un alto nivel de competencias digitales.

\section{Método}

La metodología que elegí para cumplir con dicho objetivo es la metasintesis, también llamada metaanálisis cualitativo (Bondas \& Hall, 2007a, Sandelowski \& Barroso, 2003). Dado que hay una enorme ambigüedad en el uso y la interpretación de la metasíntesis o de sus diferentes variaciones -metaresumen, síntesis temática, metaestudio, etc.--, ${ }^{1}$ así como también de ésta frente a su equivalente en el paradigma cuantitativo -el metaanálisis-, es preciso que aclaremos de qué manera la entiendo en estas líneas. En mi opinión, la metasíntesis es un método de análisis cualitativo que reúne investigaciones previas sobre un tema particular a fin de crear una interpretación nueva, integrada y exhaustiva de éstas, la cual permite una comprensión profunda del campo de estudio; de esta manera, más que un mero resumen, pretende ir más allá -lo que se expresa por el uso del prefijo 'meta'-. Por otra parte, también busco aclarar las inconsistencias encontradas, articular las diferentes concepciones teóricas y llegar a mayores niveles de abstracción, incluyendo además un elemento crítico (Bondas \& Hall, 2007a, Timulak, 2009) ${ }^{2}$.

Frente a lo anterior, la apropiación que hago de esta metodología presenta una importante diferencia frente a los modelos tradicionales de su desarrollo: la versión más usual de las metasíntesis implica una revisión cualitativa de estudios dentro del mismo paradigma (Walsh \& Downe, 2005, Timulak, 2009); por su parte, mi análisis tiene la temática estudiada como su principal fundamento, de manera que cubre tanto investigaciones de corte cuantitativo,

1 Para una revisión de las diferentes variaciones, véanse BarnettPage y Thomas (2009), Finlayson y Dixon (2008).

2 Por las anteriores razones este trabajo no deber ser considerado o entendido como una revisión de literatura, la cual, aunque a veces se emplee como un sinónimo de la metasíntesis, no lo es en realidad. Es más, si deseáramos ser aún más precisos, las características de mi investigación se pueden clasificar como un metaestudio. Para la aclaración de sus diferencias véanse Bondas y Hall (2007a) y Zhao (1991). como cualitativo y mixtas, teniendo una mirada que también podríamos considerar como mixta. Esto significa que, aunque el grueso del análisis sea cualitativo, ello no implica, como veremos más adelante, que dejemos de lado análisis estadísticos en la descripción y examen de la muestra. Esta decisión simplemente se debe a que no considero que los dos paradigmas sean posturas antagónicas o bandos en los que hay que tomar partido, sino proveedores de herramientas que pueden y deben ser empleadas según las necesidades de una investigación.

Pero antes de continuar con la descripción del estudio, es necesario realizar una aclaración adicional frente a su naturaleza y justificación. Sobre la temática de los nativos digitales, se han desarrollado -hasta donde he podido determinar- dos investigaciones previas con un acercamiento metodológico similar, ya que son revisiones sistemáticas de la literatura: la de Cabra-Torres y Marciales-Vivas (2009) y la de Esteve, Duch y Gisbert (2014). De manera que la pregunta sería, ¿para qué realizar una nueva síntesis? Tres razones justifican mi decisión: en primer lugar, y como veremos con mayor precisión más adelante, en los últimos cinco años se han multiplicado los estudios sobre el tema de los nativos digitales, de forma tal que considero importante revisar los resultados de estos nuevos estudios ${ }^{3}$; en segundo lugar, mi investigación, en tanto metasíntesis, tiene una naturaleza y unos objetivos diferentes a los de una revisión sistemática, de manera tal que reviso distintas investigaciones a las examinadas por aquellas; y, en tercer lugar, cabría esperar que haya habido cambios en este último lustro dado el aumento de la posesión de dispositivos digitales. Ahora, ello de ninguna manera quiere decir que esté dejando de lado las conclusiones y aportes de estos dos estudios; por el contrario, los considero vitales para esta empresa.

\subsection{Descripción del estudio}

De acuerdo con los objetivos de esta investigación, empleé cuatro criterios de

3 Las dos revisiones sistemáticas mencionadas cubren lo publicado hasta el 2010. 
inclusión durante el proceso de selección de los artículos: primero, que fueran reportes de investigaciones -ya fuese en inglés o españolevaluadas por pares; segundo, que sus objetivos se dirigieran al análisis de las competencias digitales $-\mathrm{o}$ de sus constructos paralelos- de los jóvenes y las jóvenes; tercero, que fueran posteriores al planteamiento original de Prensky (2001); y cuarto, que presentaran resultados empíricos y no fueran exclusivamente revisiones teóricas.

Siguiendo los criterios - de alguna manera tradicionales- de la metasíntesis (Bondas \& Hall, 2007b), excluí reportes de investigaciones presentados en libros, tesis, memorias de conferencias y cualquier forma de literatura gris, dadas las dificultades de acceder a este tipo de documentos. Asimismo, prescindí de estudios que centraran su evaluación en competencias demasiado particulares -por ejemplo, el manejo de teléfonos celulares o redes sociales-. Finalmente, y a diferencia de otras metasíntesis (Finlayson \& Dixon, 2008), no consideré la calidad de los estudios como un criterio de exclusión ${ }^{4}$.

El primer proceso fue la elección de las palabras clave para la búsqueda; para ello tomé como referencia la revisión bibliográfica previa que hice, así como el análisis de Esteve, Duch y Gisbert (2014); esto me llevó a identificar dos grupos principales de palabras clave: aquellas que marcaban los principales constructos paralelos al de los nativos digitales ${ }^{5}$ y aquellas referidas a los constructos paralelos al de las competencias digitales ${ }^{6}$.

Con ellas, establecí dos cadenas de términos -una por cada idioma-, buscando obtener una alta sensibilidad en la búsqueda, es decir, alcanzar la mayor cantidad de resultados relevantes, aun si su precisión -la cantidad de

$4 \quad$ Solo uno de ellos fue excluido por cuestiones que podríamos encuadrar en términos de calidad; pero fue más por no contar con los elementos mínimos que deseaba analizar -descripción teórica, objetivo, etc.-.

5 Generación net, generación Google, generación digital, millennials, estudiantes digitales, jóvenes digitales y homo zappiens.

6 Habilidades TIC/digitales/tecnológicas/del siglo 21/de Internet, alfabetismo computacional/digital/informacional, competencias informacionales/TIC. artículos que cumplieran con los criterios de inclusión- no fuera la mejor (Ring, Ritchie, Mandava \& Jepson, 2010, p. 11). La estructura general de las cadenas $^{7}$ fue adaptada a las características de los motores de búsqueda de cada base de datos, dado que no todos, por ejemplo, permiten el uso de operadores booleanos o difieren en la forma de manejar las búsquedas de los plurales. Las cadenas, siempre que fue posible, fueron aplicadas a las opciones de título, resumen y palabras clave, en el rango temporal del año 2001 al 2016.

Las bases de datos y servicios de indexación en las que apliqué la búsqueda fueron: Scopus, Science Direct, SciELO, Web of Science, Redalyc, Google Scholar, Doaj y Ebsco, cuyos resultados en términos de número de artículos potencialmente relevantes (tested positive; Shaw et al., 2004) y aquellos que establecí como efectivamente relevantes (diagnosed positive; Shaw et al., 2004) pueden observarse en la figura 1 -la cual «tomé prestada» de los estándares para la realización de los metaanálisis-. Dicha relevancia la establecí al analizar sus títulos y palabras clave.
Por ejemplo, para el caso del inglés, la estructura de la cadena fue: ("Digital Competenc*" OR "ICT skill*" OR "information technology skill*" OR "digital skill*” OR "computer Literacy" OR "IT literacy" OR "Information technology literacy" OR "Information Literacy" OR "Media Literacy" OR "Digital literacy" OR "technology skill*" OR "21st century skill*" OR "Literacy skill* for the twenty-first century" OR "Internet skill*") AND ("Digital nativ*" OR "net generation" OR "Google generation" OR "Digital generation" OR "Millennials" OR "Young" OR "homo zappiens"). 
Figura 1. Proceso de selección de los artículos.

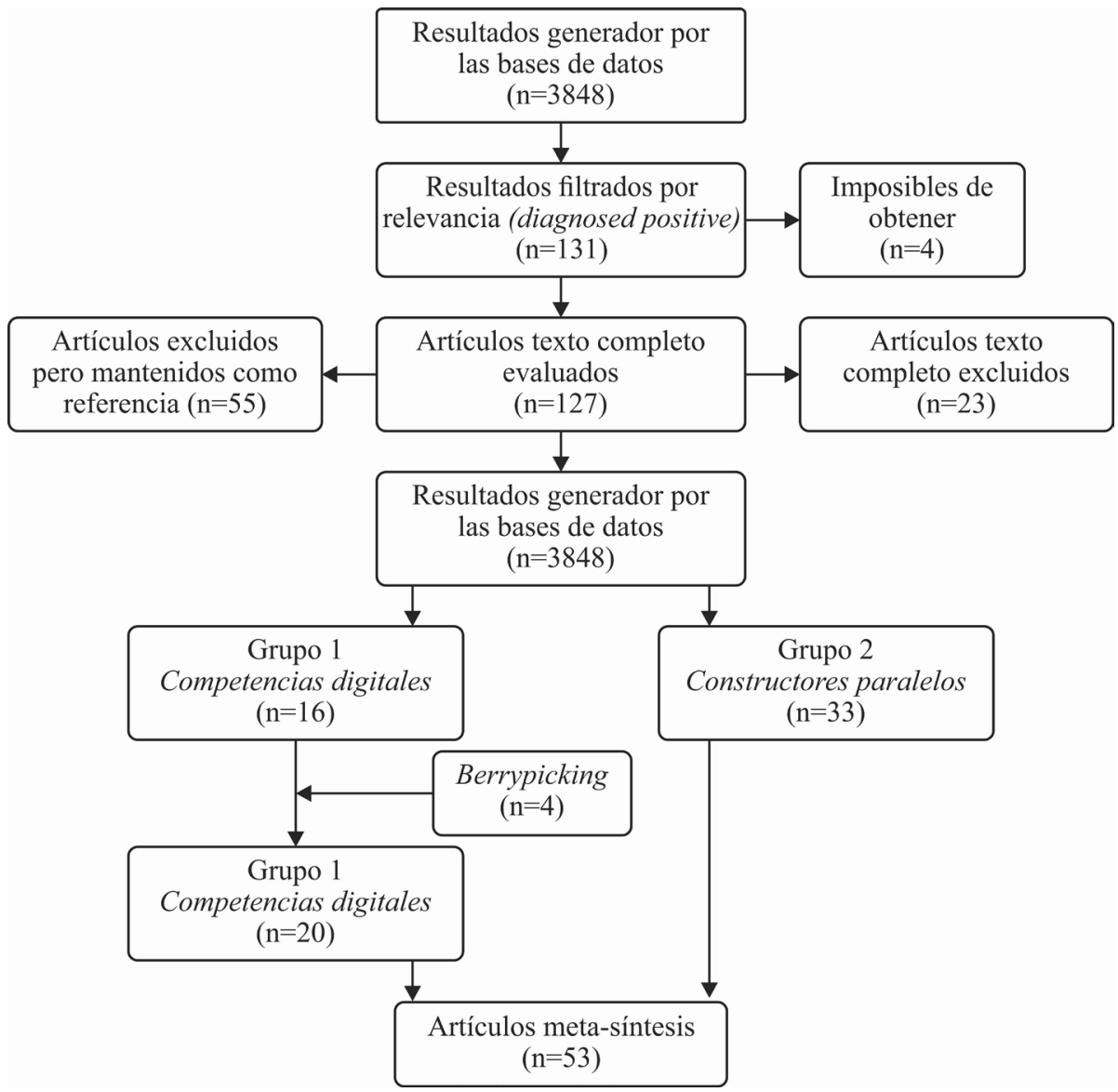

Nota: adaptado de los estándares Prisma para el desarrollo de meta-análisis. Moher, D., Liberati, A., Tetzlaff, J. \& Altman, D. G., The Prisma Group (2009). Preferred Reporting Items for Systematic Reviews and Meta-Analyses: The Prisma Statement. PLoS Med, 6 (7), e1000097. Doi:10.1371/journal.pmed1000097 Licencia Creative Commons CC BY 2.0

En una segunda fase, de los 131 artículos resultantes revisé sus abstracts -cuando éstos no contenían la información completa, revisé ciertas partes clave de los textos-, a fin de determinar que efectivamente cumplieran con mis criterios de inclusión y no existieran artículos duplicados. Esta evaluación me permitió establecer los 49 artículos que examinaría, los 55 que guardaría como referencia y, por último, los 23 excluidos.

Durante el desarrollo de este proceso decidí dividir los 49 artículos en dos categorías: la de los que presentaran estudios que hubiesen evaluado expresamente competencias digitales -o su análogo directo, las competencias TIC-; mientras que la segunda contendría artículos que evaluaran constructos paralelos $-v . g$. las habilidades digitales- y que llegaran a conclusiones sobre la existencia de los nativos digitales. La razón de este agrupamiento es simple: todos y cada uno de ellos proporcionan pistas frente a mi pregunta de investigación, mas, por su naturaleza misma, no podrían ser analizados de manera estructurada como una unidad.

Esta división también me permite realizar una importante aclaración sobre el presente documento: éste cubre la primera fase de análisis única y exclusivamente del primer grupo de artículos, el cual consta de los 20 artículos relacionados en la tabla $1 .{ }^{8}$

8 Todas las tablas de este documento son elaboración del autor. 
Tabla 1. Artículos incluidos en la metasíntesis.

\begin{tabular}{|l|c|l|c|}
\hline \multicolumn{1}{|c|}{ Autor(es) } & Año & \multicolumn{1}{|c|}{ Autor(es) } & Año \\
\hline Aesaert, K., \& van Braak, J. & 2015 & $\begin{array}{l}\text { Hatlevik, O. E., Guomundosdóttir, G. B., \& } \\
\text { Loi, M. }\end{array}$ & 2015 \\
\hline Arias, M., Torres, T., \& Yánez, J. C. & 2014 & Li, Y., \& Ranieri, M. & 2010 \\
\hline $\begin{array}{l}\text { Arras, A. M., Torres-Gastelú, C. A., \& García- } \\
\text { Valcárcel, A. }\end{array}$ & 2011 & Matamala, C. & 2014 \\
\hline Brazo, L., Ipiña, N., \& Zuberogoitia, A. & 2011 & Pino, M., \& Soto, J. & 2010 \\
\hline Calvani, A., Fini, A., \& Ranierí, M. & 2010 & Roig, R., \& Pascual, A. M. & 2012 \\
\hline Calvani, A., Fini, A., Ranierí, M., \& Picci, P. & 2012 & Romaniuk, M. W. & 2015 \\
\hline Carrasco, M. E. E., Sánchez, C., \& Carro, A. & 2015 & Romero, M., \& Minelli, J. & 2011 \\
\hline Centeno, G., \& Cubo, S. & 2013 & San Nicolás, M. B., Fariña, E., \& Area, M. & 2012 \\
\hline $\begin{array}{l}\text { García, J., García-Sánchez, J. N., Álvarez- } \\
\text { Fernández, M. L., \& Díez-Caso, H. }\end{array}$ & 2014 & Torres-Gastelú, C. A. & 2015 \\
\hline Guo, R. X., Dobson, T., \& Petrina, S. & 2008 & $\begin{array}{l}\text { Torres-Gastelú, C. A., Domínguez, A. L., } \\
\text { Flores, M. A., Kiss, G., \& Alejandre, A. R. }\end{array}$ & 2015 \\
\hline
\end{tabular}

Finalmente, aunque las metasíntesis no buscan determinar la «verdadera» o «completa» población, ya que esto requeriría un estándar para la búsqueda de evidencia cualitativa que no existe (Shaw et al., 2004), de todas formas continué con la búsqueda de nuevos artículos relevantes durante la lectura y el análisis de los textos. Para ello, empleé la técnica del berrypicking (Bates, 1989); esto es, analicé las citas y fuentes de cada artículo buscando nuevas fuentes potenciales de información. Por medio de este procedimiento, logré identificar cuatro nuevos artículos, lo que me indicó que mi búsqueda inicial había sido bastante cercana a esa esquiva población.

\subsection{Estrategias de análisis}

Para el análisis de los datos seguí las directrices de Miles, Huberman y Saldaña (2014) para el análisis de casos múltiples. Las conclusiones parciales que presento en este escrito se derivan de la estrategia del amontonamiento (stacking) de casos comparables. Para ello creé una matriz del tipo resumen analítico de contenido ${ }^{9}$, en la

9 Content-analytic summary matrix. Es una matriz que agrupa to-

Rev.latinoam.cienc.soc.niñez juv 15 (1): 471-489, 2017 http://revistalatinoamericanaumanizales.cinde.org.co DOI: 10.11600/1692715x.1513014062016 que condensé la información, de manera que me permitiera realizar una comparación exploratoria de los diferentes estudios. Dicha información analizada y organizada fue: año de publicación, paradigma, pregunta/hipótesis de la investigación, objetivo, concepción de competencia digital, método, herramienta de evaluación, muestra, principales resultados y nivel de competencias digitales demostrado por los participantes. Posteriormente generé metamatrices descriptivas ordenadas por caso $^{10}$ para los tres elementos principales: concepción, método y resultados, de forma que pudiese comparar y analizar los diferentes casos a partir de dichos elementos.

\section{Resultados y discusión}

Los 20 estudios que cubro en este análisis representan trabajos desarrollados en cuatro continentes, pero con una especial

dos los datos relevantes de varios casos en una única forma para su análisis exploratorio (Miles, Huberman \& Saldaña, 2014, p. 148).

10 Case-Ordered Descriptive Meta-Matrix. Aquella que contiene los datos de todos los casos organizados según una variable, permitiendo así ver sus diferencias y similitudes (Miles, Huberman \& Saldaña, 2014, p. 214). 
presencia de los realizados en España (55\%) y México (20\%). También tienen una sugerente representación aquellos cuya población de estudio son estudiantes de licenciatura o de maestría en educación (45\%), lo que responde, según estos textos, a la creciente necesidad de que los futuros docentes cuenten con este tipo de competencias. Por otra parte, como lo mencioné anteriormente, es notorio cómo se han multiplicado los estudios sobre las competencias digitales en el último lustro, de tal manera que sólo el $20 \%$ de los artículos cubiertos fueron publicados previamente al año 2011, como podemos observar en la tabla 2.

Tabla 2. Artículos por año.

\begin{tabular}{|c|c|c|}
\hline Año & $\mathbf{f}$ & $\mathbf{\%}$ \\
\hline 2008 & 1 & 5 \\
\hline 2010 & 3 & 15 \\
\hline 2011 & 3 & 15 \\
\hline 2012 & 3 & 15 \\
\hline 2013 & 1 & 5 \\
\hline 2014 & 3 & 15 \\
\hline 2015 & 6 & 30 \\
\hline & $\mathbf{2 0}$ & $\mathbf{1 0 0}$ \\
\hline
\end{tabular}

\subsection{Concepciones y teorizaciones sobre la competencia digital}

Respecto a la concepción de competencia digital, mi primera conclusión -detectada desde la revisión previa de los artículos- es que, al menos dentro del sub-grupo de estudios analizados, las expresiones 'competencia digital' y 'competencia en TIC son empleadas como sinónimos. Esto no quiere decir que necesariamente los investigadores e investigadoras hayan establecido expresamente dicha equivalencia, pero en sus marcos teóricos no hay diferencias entre una y otra. Por ejemplo, Torres-Gastelú (2015) presenta la competencia en TIC como un «grupo de habilidades, conocimientos y actitudes aplicados a la utilización de sistemas de información y comunicación» (p. 146), mientras que Centeno y Cubo (2013) hablan de la competencia digital en términos de «la adquisición de conocimientos, destrezas y actitudes basadas en el uso elemental del hardware de los ordenadores, sus sistemas operativos, el software como herramienta de trabajo...» (p. 521). Como podemos observar, ambas definiciones son completamente simétricas.

El segundo patrón que pude establecer es que todas las concepciones del término, de una y otra forma, se centran en los mismos elementos nucleares: los conocimientos -el saber qué-, las habilidades -el saber cómo-, las actitudes -positivas- y los usos eficaces y éticos de la tecnología. ${ }^{11}$ De forma más precisa, los estudios pueden ser divididos en tres grupos según el énfasis que realicen en ciertas combinaciones de los anteriores elementos: en el primero se encuentran las que entienden la competencia digital como la suma de conocimientos, habilidades y actitudes dirigidas al ámbito de lo tecnológico. Si bien no se lo exprese de esta manera, en este caso creo ver la definición general de competencia defendida por la OECD (Rychen \& Salganik, 2001), siendo simplemente trasladada a lo digital. En el segundo grupo podemos reunir aquellas que ponen el acento en la praxis; es decir, subrayan el uso productivo, exitoso y ético de la tecnología, pero no en un sentido en el que la tecnología es el fin último, sino de manera que se la entiende como una herramienta que permite a las personas desarrollarse y expresarse en un mundo cada vez más mediado por lo digital.

Finalmente, y como un elemento digno de ser subrayado, el tercer grupo está constituido por siete investigaciones que no expresan ningún concepto de competencia o no se adhieren explícitamente a ninguno previo. Esto es importante ya que, en un campo con tan pocos acuerdos conceptuales y tantos constructos que «compiten» unos con otros, es muy llamativo

11 A lo que podríamos añadir el saber cuándo y el saber dónde para el logro de la transferencia de dichos conocimientos y habilidades (Acosta-Silva \& Vasco, 2013). 
que los investigadores e investigadoras no tratemos de colaborar en la clarificación de la situación o, al menos, pongamos nuestra apuesta frente a alguno de ellos. Y es precisamente en este contexto en donde dos trabajos analizados se destacan por darse a la tarea de tratar de explorar el constructo, a fin de aclararlo y presentar propuestas fundamentadas tanto teórica como empíricamente. Por un lado, está el grupo de investigación de Calvani, Fini y Ranieri -representado en tres investigaciones de esta muestra- $y$, por otro, la investigación de Aesaert y van Braak (2015).

\subsection{Métodos e instrumentos}

Al revisar los métodos reportados por los artículos, el primer patrón observable es el notorio «dominio» del paradigma cuantitativo en los estudios de la muestra (tabla 3). Seguramente se podrá argumentar que, como estamos frente a investigaciones con metas evaluativas, lo cuantitativo presenta las herramientas clásicas para enfrentarlas. Sin embargo, con la misma fuerza se podría argumentar que la complejidad del fenómeno y sus marcadas características socio-culturales llaman también al empleo de lo cualitativo para su exploración.

Ahora, y precisamente hablando de lo cualitativo, también es llamativo cómo, en los pocos estudios que lo aplican, su inclusión en todos los casos se limita al empleo de grupos focales. Obviamente, esta elección no tiene nada censurable. Sin embargo, al menos en los artículos revisados, tristemente no se reporta ningún análisis cualitativo de los resultados de dichos grupos focales; es decir, los autores y autoras terminan solamente empleándolos como una fuente para presentar extractos de los comentarios de los participantes. De nuevo, el «darle la palabra» a quienes participan es una característica fundamental de dicho paradigma, pero la ausencia de los resultados de los análisis cualitativos se me antoja como una deuda de estos artículos. 
Tabla 3. Paradigmas e instrumentos reportados.

\begin{tabular}{|c|c|c|c|}
\hline Autor(es) & Paradigma & Instrumento & Origen del Instrumento \\
\hline Arias, Torres, \& Yánez & \multirow{17}{*}{ Cuantitativo } & \multirow{4}{*}{ Escala de Likert } & $\begin{array}{l}\text { Específicamente diseñado } \\
\text { para el estudio }\end{array}$ \\
\hline Centeno, G., \& Cubo & & & Basado en Incotic \\
\hline Roig \& Pascual & & & $\begin{array}{c}\text { Cuestionario de Guzmán } \\
\text { (2008) }\end{array}$ \\
\hline Romero \& Minelli & & & Adaptación de Romero (2008) \\
\hline Carrasco, Sánchez, \& Caro & & Cuestionario y Escala de Likert & $\begin{array}{l}\text { Específicamente diseñado } \\
\text { para el estudio }\end{array}$ \\
\hline $\begin{array}{l}\text { García, García-Sánchez, } \\
\text { Álvarez-Fernández, \& Díez- } \\
\text { Caso }\end{array}$ & & \multirow{4}{*}{ Cuestionario } & $\begin{array}{l}\text { Específicamente diseñado } \\
\text { para el estudio }\end{array}$ \\
\hline Romaniuk & & & $\begin{array}{l}\text { Específicamente diseñado } \\
\text { para el estudio }\end{array}$ \\
\hline San Nicolás, Fariña, \& Area & & & $\begin{array}{l}\text { Específicamente diseñado } \\
\text { para el estudio }\end{array}$ \\
\hline $\begin{array}{l}\text { Torres-Gastelú, Domínguez, } \\
\text { Flores, Kiss, \& Alejandre }\end{array}$ & & & $\begin{array}{l}\text { Específicamente diseñado } \\
\text { para el estudio }\end{array}$ \\
\hline $\begin{array}{l}\text { Arras, Torres-Gastelú, \& } \\
\text { García-Valcárcel }\end{array}$ & & Encuesta & $\begin{array}{l}\text { Específicamente diseñado } \\
\text { para el estudio }\end{array}$ \\
\hline Matamala & & \multirow{2}{*}{ Prueba } & $\begin{array}{l}\text { Trabaja con datos secundarios } \\
\text { para el estudio }\end{array}$ \\
\hline Aesaert \& van Braak & & & $\begin{array}{l}\text { Específicamente diseñado } \\
\text { para el estudio }\end{array}$ \\
\hline Calvani, Fini, \& Ranieri & & \multirow{3}{*}{ Prueba de Opción Múltiple } & \multirow{3}{*}{$\begin{array}{l}\text { Instant Digital Competence } \\
\text { Assessment (iDCA) }\end{array}$} \\
\hline Calvani, fini, Ranieri, \& Picci & & & \\
\hline Li \& Ranieri & & & \\
\hline \multirow{2}{*}{$\begin{array}{l}\text { Hatlevik, Guomundsdóttir, \& } \\
\text { Loi }\end{array}$} & & Cuestionario & \multirow{2}{*}{$\begin{array}{l}\text { Específicamente diseñado } \\
\text { para el estudio }\end{array}$} \\
\hline & & Prueba de Opción Múltiple & \\
\hline \multirow[t]{2}{*}{ Brazo, Ipiña, \& Zuberogoitia } & \multirow{8}{*}{ Mixto } & Cuestionario & $\begin{array}{l}\text { Adaptación de Kennedy } \\
\text { (2009) }\end{array}$ \\
\hline & & Grupo Focal & ----- \\
\hline \multirow[t]{2}{*}{ Guo, Dobson, \& Petrina } & & Cuestionario & $\begin{array}{l}\text { Adaptación de Gable \& Wolf } \\
\text { (1993); Gibson \& Nocente } \\
\text { (1998); ISTE NETS (2000); } \\
\text { Scheffler \& Logan (1999) }\end{array}$ \\
\hline & & Observaciones & ----- \\
\hline \multirow[t]{2}{*}{ Pino \& Soto } & & Cuestionario & $\begin{array}{l}\text { Adaptación de Gallego } \\
(2007), \text { Mominó y Sigalés } \\
(2009)\end{array}$ \\
\hline & & Grupo Focal & ---- \\
\hline \multirow[t]{2}{*}{ Torres-Gastelú } & & Cuestionario & $\begin{array}{l}\text { Específicamente diseñado } \\
\text { para el estudio }\end{array}$ \\
\hline & & Grupo Focal & ----- \\
\hline
\end{tabular}


El siguiente elemento que se destaca en los métodos de evaluación es cómo la mitad de los estudios emplea instrumentos específicamente diseñados por y para ellos (tabla 3). La lectura que hago de este fenómeno es que ello se deriva de la falta de acuerdos -y de preocupaciónsobre qué es o qué son la(s) competencia(s) digital(es) y cuáles son sus componentes. Así que -al menos dentro de la muestra analizadalos investigadores e investigadoras terminen proponiendo sus propias lecturas sobre las competencias, sus propias dimensiones a considerar y sus propios comportamientos a evaluar, teniendo como consecuencia que sea prácticamente imposible hacer comparaciones totalmente transversales de sus resultados - si bien existen solapamientos-.

Respecto a los instrumentos empleados, es patente la homogeneidad de su naturaleza. El $75 \%$ de los estudios emplean herramientas -ya sean cuestionarios o escalas de Likert-diseñadas para que los estudiantes y las estudiantes evalúen sus competencias de forma autoevaluativa y retrospectiva (tabla 4$)^{12}$. Este es el resultado que más me interesa subrayar, sobre todo porque mucho de lo que se pueda concluir de una investigación depende de sus instrumentos de recolección de datos. Considero que si mi objetivo es determinar las competencias, las autoevaluaciones retrospectivas no son las herramientas más potentes. Sus limitaciones han sido ampliamente establecidas: principalmente, los problemas de validez generados por el sesgo de la percepción subjetiva (self-reported bias) que es particularmente fuerte en las personas adolescentes (Fan et al., 2006); asimismo, las autoevaluaciones solo presentan datos indirectos (Litt, 2013). De manera tal que, si mi objetivo es establecer las percepciones de los jóvenes sobre sus competencias, las autoevaluaciones son la herramienta adecuada, con la que -sin duda- podré encontrar datos importantes en la búsqueda de falencias y en el desarrollo de intervenciones educativas para darles solución.

12 Lo cual también se puede observar en los estudios que evalúan constructos paralelos como los de las habilidades digitales y la alfabet Sin información ización digital (Gui \& Argentin, 2011)

Rev.latinoam.cienc.soc.niñez juv 15 (1): 471-489, 2017 http://revistalatinoamericanaumanizales.cinde.org.co DOI: $10.11600 / 1692715 x .1513014062016$
Pero si quiero evaluar sus competencias, lo ideal sería trabajar con mediciones directas, como las pruebas de desempeño (Aesaert \& van Braak, 2015). 
Tabla 4. Paradigmas e instrumentos reportados.

\begin{tabular}{|c|c|c|c|}
\hline Autor(es) & $\begin{array}{c}\text { Forma de } \\
\text { evaluación }\end{array}$ & Muestra & Rangos Etáreos \\
\hline Arias, Torres, \& Yánez & \multirow{19}{*}{ Autoevaluación } & $\begin{array}{l}578 \text { estudiantes universitarios } \\
\text { españoles }\end{array}$ & Sin información \\
\hline Centeno, G., \& Cubo & & $\begin{array}{l}101 \text { estudiantes universitarios } \\
\text { españoles }\end{array}$ & Sin información \\
\hline Roig \& Pascual & & $\begin{array}{l}61 \text { estudiantes españolas (todas } \\
\text { mujeres) }\end{array}$ & 19 a 44 años (media 22) \\
\hline Romero \& Minelli & & 54 estudiantes universitarios españoles & \begin{tabular}{l}
\multicolumn{1}{c}{21 a 43 años: } \\
a) nacidos antes de 1982 \\
b) nacidos después de \\
1982 \\
\end{tabular} \\
\hline $\begin{array}{l}\text { Carrasco, Sánchez, \& } \\
\text { Carro }\end{array}$ & & 15 estudiantes universitarios españoles & $\begin{array}{l}\text { a) } 20-29(53 \%) \\
\text { b) } 30-49(47 \%) \\
\end{array}$ \\
\hline $\begin{array}{l}\text { García, García-Sánchez, } \\
\text { Álvarez-Fernández, \& } \\
\text { Díez-Caso }\end{array}$ & & $\begin{array}{l}286 \text { estudiantes universitarios } \\
\text { españoles }\end{array}$ & $\begin{array}{l}\text { a) menos } 22(61) \\
\text { b) 22-23 (69) } \\
\text { c) } 24-26(71) \\
\text { d) 26-35 (54) } \\
\text { e) mayores } 36(31) \\
\end{array}$ \\
\hline Romaniuk & & $\begin{array}{l}135 \text { estudiantes universitarios } \\
\text { españoles }\end{array}$ & 19 a 22 años \\
\hline $\begin{array}{l}\text { San Nicolás, Fariña, \& } \\
\text { Area }\end{array}$ & & $\begin{array}{l}1561 \text { alumnos españoles universitarios } \\
206 \text { profesores }\end{array}$ & Sin información \\
\hline $\begin{array}{l}\text { Torres-Gastelú, } \\
\text { Domínguez, Flores, Kiss, } \\
\& \text { Alejandre }\end{array}$ & & 979 estudiantes secundaria mexicanos & Sin información \\
\hline $\begin{array}{l}\text { Arras, Torres-Gastelú, \& } \\
\text { García-Valcárcel }\end{array}$ & & $\begin{array}{l}1710 \text { estudiantes universitarios } \\
\text { españoles y mexicanos }\end{array}$ & Sin información \\
\hline Matamala & & $\begin{array}{c}4496 \text { estudiantes chilenos de } \\
\text { secundaria }\end{array}$ & Sin información \\
\hline \multirow{2}{*}{$\begin{array}{l}\text { Brazo, Ipiña, \& } \\
\text { Zuberogoitia }\end{array}$} & & $\begin{array}{l}249 \text { estudiantes españoles } \\
\text { universitarios }\end{array}$ & Promedio 20 años \\
\hline & & 9 estudiantes españoles universitarios & Sin información \\
\hline \multirow[t]{2}{*}{ Guo, Dobson, \& Petrina } & & $\begin{array}{c}2583 \text { estudiantes de especialización } \\
\text { (Postbaccalaureate) canadienses y } \\
\text { estadounidenses }\end{array}$ & $\begin{array}{l}\text { a) } 20-24(46 \%) \\
\text { b) } 25-29(37 \%) \\
\text { c) } 30-40(10 \%) \\
\text { d) más de } 40(3 \%) \\
\end{array}$ \\
\hline & & Sin información & Sin información \\
\hline \multirow{2}{*}{ Pino \& Soto } & & $\begin{array}{l}219 \text { estudiantes universitarios } \\
{\text { españoles y } 10 \text { docentes }^{\mathrm{a}}}\end{array}$ & 19 a 33 años (media 21) \\
\hline & & $\begin{array}{l}5 \text { estudiantes universitarios españoles y } \\
3 \text { docentes }^{\mathrm{b}}\end{array}$ & Sin información \\
\hline \multirow{2}{*}{ Torres-Gastelú } & & $\begin{array}{l}308 \text { estudiantes mexicanos } \\
\text { universitarios }^{\mathrm{a}}\end{array}$ & \multirow{2}{*}{ Sin información } \\
\hline & & $\begin{array}{c}40 \text { estudiantes mexicanos } \\
\text { universitarios }\end{array}$ & \\
\hline $\begin{array}{l}\text { Hatlevik, Guomundsdóttir, } \\
\text { \& Loi }\end{array}$ & $\begin{array}{l}\text { Autoevaluación } \\
\text { y desempeño }\end{array}$ & 593 estudiantes de secundaria noruega & 17 a 18 años \\
\hline Aesaert \& van Braak & \multirow{4}{*}{ Desempeño } & 378 estudiantes de primaria belgas & 10 a 13 años (media 12) \\
\hline Calvani, Fini, \& Ranieri & & \multicolumn{2}{|c|}{$\begin{array}{c}\text { Presentan los resultados de los otros dos artículos de los mismos } \\
\text { autores en Italia y China }\end{array}$} \\
\hline $\begin{array}{l}\text { Calvani, Fini, Ranieri, \& } \\
\text { Picci }\end{array}$ & & 1056 jóvenes italianos & 14 a 16 años (media 15$)$ \\
\hline Li \& Ranieri & & 317 estudiantes de secundaria chinos & 14 a 17 años \\
\hline
\end{tabular}


Sin embargo, y este es el mayor descuido que veo en una importante cantidad de estudios de esta muestra -7 de 20-, estos caen en el error de igualar estas percepciones con las actuales competencias de los jóvenes, lo que implica que se llega a conclusiones sobre competencias a partir de las opiniones y autoevaluaciones que los participantes hacen de su propio desempeño ${ }^{13}$.

Por otra parte, la verdad es que, aunque sean complejas, sí es posible realizar evaluaciones de desempeño con un alto nivel de validez ecológica. ${ }^{14}$ Un ejemplo concreto de ello se encuentra en esta muestra: Aesaert y van Braak (2015) diseñaron una prueba en la que los sujetos participantes debían realizar una serie de tareas que poseían las mismas características de aquellas que dichos sujetos podrían llegar a encontrar en su vida diaria, de forma tal que demostrasen tanto sus conocimientos como sus habilidades de forma directa ${ }^{15}$.

Finalmente, no puedo dejar de notar cómo la mitad de los artículos (tabla 4) no reportan una variable fundamental en los estudios sobre jóvenes: la edad de sus participantes. Podría suponer que este patrón es simplemente una característica circunstancial de la muestra, pero infortunadamente debo reportar otros errores metodológicos en varios artículos: inconsistencias en la presentación de la información, incoherencia entre los objetivos y las conclusiones presentadas y ausencia de los procesos de validación de los instrumentos.

\subsection{Análisis de los resultados}

Como lo acabo de plantear, es imposible hacer una comparación de los resultados de los estudios, dada la fuerte variación de las dimensiones y elementos medidos por cada uno de ellos, de manera que solo me limitaré a explorar qué se plantea frente a la pregunta

13 El problema es tan extendido que sólo uno de todos los estudios que emplean autoevaluaciones (Romero \& Minelli, 2011) explícitamente es consciente de las limitaciones de éstas, y sus conclusiones mantienen coherencia con ello.

14 Véase Tupper y Cicerone (1990).

15 Para otro ejemplo, aunque en el ámbito de la evaluación de la alfabetización digital, véase Eshet-Alkali y Amichai-Hamburger (2004). central. Según los resultados reportados, ¿es posible mantener las afirmaciones de Prensky sobre las competencias digitales sofisticadas y extendidas entre los jóvenes? En la tabla 5, podemos observar cómo el $80 \%$ de los estudios las ponen en entredicho: para un $25 \%$ la respuesta es un contundente no; simplemente los jóvenes fallan en demostrar - $\mathrm{O}$ en creer que tienen- un alto nivel de competencias y, en el mejor de los casos, a éstas apenas se las considera como moderadas (v. g. Carrasco, Sánchez \& Carro, 2015). Resultados similares también se encuentran en la literatura (por ejemplo, Thinyane, 2010). Para otro grupo de estudios, aunque la respuesta en general continúa siendo un no, reconocen que no se puede negar que entre los jóvenes sí existen muchos con un alto nivel de competencias, aunque sean una minoría -lo cual también ha sido reportado en los modelos alternos; v. g., Kennedy et al., 2008-. De manera similar, están quienes detectan una alta fluctuación entre las competencias de los participantes, tanto de forma vertical en una única dimensión -por ejemplo, la búsqueda de información-, como de forma horizontal al comparar todas las dimensiones evaluadas, lo que de nuevo va en contra de clasificarlos a todos como competentes; ello está también reportado en otras investigaciones fuera de esta muestra (entre otras, Helsper \& Eynon, 2010, Zimic, 2009). En este caso, conviene anotar que las actividades en las que se demuestra competencia generalmente están dirigidas a las comunicaciones interpersonales y al ocio, mientras que sus mayores dificultades están en el análisis de la calidad de la información, lo que es coherente con otras investigaciones de la literatura que revisan constructos paralelos $(v$. g. Eshet-Alkali \& Amichai-Hamburger, 2004).

Por otra parte, sólo hay tres estudios que llegan a conclusiones opuestas a lo anterior los cuales considero «refutacionales» dentro de la terminología de la metasíntesis [Timulak, 2009]-, esto es, que sostienen que sus participantes sí son competentes digitales y, por lo tanto, Prensky tenía razón -aunque sólo uno de ellos lo expresa explícitamente así-. Estos casos comparten tres características: en primer lugar, los tres son investigaciones desarrolladas 
con estudiantes de universidades españolas; segundo, están basados en autoevaluaciones; $\mathrm{y}$, tercero, sus instrumentos fueron diseñados específicamente para dichos estudios. Pero determinar el porqué de sus resultados refutacionales está fuera del alcance de este examen, al menos por el momento.

Tabla 5. Resultados reportados en relación a la competencia digital.

\begin{tabular}{|c|c|c|c|}
\hline $\begin{array}{l}\text { ¿Son competencias } \\
\text { digitales? }\end{array}$ & Estudios & $\mathbf{f}$ & $\%$ \\
\hline $\mathrm{Si}$ & $\begin{array}{l}\text { Arias, Torres, \& Yánez (2014); García, García-Sánchez, Álvarez- } \\
\text { Fernández, \& Díez-Caso (2014); Roig \& Pascual (2012 }\end{array}$ & 3 & 15 \\
\hline No & $\begin{array}{l}\text { Carrasco, Sánchez, \& Carro (2015); Romaniuk (2015); Romero \& Minelli } \\
\text { (2011); Torres-Gastelú, Domínguez, Flores, Kiss, \& Alejandre (2015) }\end{array}$ & 5 & 25 \\
\hline $\begin{array}{l}\text { Existen, pero son una } \\
\text { minoría }\end{array}$ & $\begin{array}{l}\text { Calvani, Fini, \& Ranieri (2010); Calvani, Fini, Ranieri, \& Picci (2012); } \\
\text { Hatlevik, Guomundsdóttir, \& Loi (2015); Li \& Ranieri (2010); Matamala } \\
\text { (2014) }\end{array}$ & 5 & 25 \\
\hline Sus competencias fluctúan & $\begin{array}{l}\text { Aesaert \& van Braak (2015); Arras, Torres-Gastelú, \& García-Valcárcel } \\
\text { (2011); Centeno, G., \& Cubo (2013); Pino \& Soto (2010); San Nicolás, } \\
\text { Fariña, \& Area (2012) }\end{array}$ & 5 & 25 \\
\hline $\begin{array}{l}\text { No son más competentes } \\
\text { que los adultos }\end{array}$ & Guo, Dobson, \& Petrina (2008) & 1 & 5 \\
\hline \multirow[t]{2}{*}{$\begin{array}{l}\text { No plantean una } \\
\text { conclusión sobre ello }\end{array}$} & Brazo, Ipiña, \& Zuberogoitia (2011) & 1 & 5 \\
\hline & & 20 & 100 \\
\hline
\end{tabular}

\section{Conclusiones}

En el presente trabajo he revisado 20 artículos publicados en los últimos 15 años que han buscado evaluar las competencias digitales de los jóvenes y las jóvenes, con el fin de establecer si sus resultados soportan las afirmaciones de Prensky en relación con las supuestas competencias generalizadas de los nativos digitales. Para ello, analicé sus concepciones, métodos y resultados. El producto de este proceso me permite afirmar que tales aseveraciones en general parecen no estar soportadas fácticamente $\mathrm{y}$, por ende, ser inducciones mal realizadas basadas en apenas un subgrupo de jóvenes. Esta conclusión es coherente con la obtenida por un estudio paralelo al mío (Cabra-Torres \& MarcialesVivas, 2009).

Sin embargo, esta conclusión debe ser matizada, a fin de no caer en el mismo problema de Prensky de crear estereotipos: en primer lugar, el que no todos los individuos jóvenes sean competentes desde luego presenta el hecho de que sí existen quienes poseen altísimas competencias o que son competentes pero únicamente en algunas de ellas -por ejemplo, aquellas referidas a los procesos de comunicación-, demostrando efectivamente un promedio de desempeño mayor al de otros rangos de edad. En segundo lugar, en general los jóvenes parecen y dicen sentirse más cómodos y familiarizados con la tecnología (Bennet, Maton $\&$ Kervin, 2008, Zimic, 2009) y es por ello que pueden darse procesos de retrosocialización (Morduchowicz, 2008) en los que los jóvenes y las jóvenes enseñan a sus mayores a utilizar elementos tecnológicos (Acosta-Silva \& Muñoz, 2012). Así las cosas, la situación que se presenta, más que la de un grupo homogéneo de jóvenes, es la de una enorme variación en sus competencias digitales. De manera que los nativos digitales parecen disolverse en una larga gradación de comportamientos.

Por otra parte, algunos estudios nos recuerdan que todavía existe una brecha digital que no permite el acceso por igual a toda la gente joven; es así que millones de sujetos jóvenes nacidos en las últimas dos décadas y media no han tenido acceso -o este es muy limitado- 
a la tecnología, y por ello no solo no pueden considerarse como nativos, sino que, de forma más preocupante, se están quedando aislados. Ello me lleva a recordar los peligros que genera esta etiqueta: por un lado, disuade a padres, madres, docentes y al Estado a tomar partido en la educación digital de las personas jóvenes ya que no se la considera importante (Guo, Dobson \& Petrina, 2008); asimismo, el que los jóvenes y las jóvenes crean ser competentes también tiene el efecto indeseable de que no consideren trascendental desarrollarlas más allá de su estado actual (Romaniuk, 2015).

Por otra parte, como cualquier otro estereotipo, la etiqueta «nativos digitales» nos hace olvidar de que más que buscar similitudes deberíamos estar preocupados por entender y hacer visible la diversidad. De la misma forma, tampoco podemos olvidar que al dividir entre competentes e in-competentes podemos caer en la misma dicotomía que entre nativos e inmigrantes.

La siguiente conclusión que puedo obtener es que, en un campo tan complejo y con tantas aristas como el de las competencias digitales, se requieren estudios con una potencia teórica y metodológica que infortunadamente no he visto en todas de las investigaciones analizadas. El principal problema que he detectado es que no se están evaluando directamente las competencias, sino la percepción que de éstas tienen los participantes, la cual, como lo he mostrado previamente, tiende a ser incorrecta. Esta situación la considero completamente insostenible: si precisamente el énfasis de las competencias está en el saber y, sobre todo, en el saber hacer, deberíamos estar evaluando cuánto saben y qué tanto son capaces de hacer, en vez de las percepciones que tengan sobre ello. De esta manera, éste se convierte en el principal problema a corregir en posteriores investigaciones.

Otro punto clave en futuros trabajos es la corrección de los errores metodológicos que detallé arriba. Más allá de fallas «estéticas», éstos se convierten en problemas que no permiten la replicación de los resultados de los estudios para el caso de los realizados bajo el paradigma cuantitativo- $y$, en general, en elementos que le restan potencia a sus conclusiones y hallazgos.
En consecuencia, los retos para próximas investigaciones en este campo son múltiples: por un lado está la cuestión de definir cuáles son las competencias digitales a considerar, o visto desde otra óptica, determinar qué considerar como un comportamiento digitalmente competente. Con ello nos deberemos enfrentar a la difícil tarea de buscar acuerdos entre la multitud de opciones que se han planteado. Este aspecto es definitivo ya que al revisar los resultados de las investigaciones examinadas podríamos aventurarnos a hipotetizar que no es que los participantes no tengan competencias digitales, sino que las que hemos medido hasta el momento no son las que ellos efectivamente tienen y valoran. Esto es, podemos establecer una ruptura entre las que esperamos que las personas jóvenes desarrollen y las que efectivamente tienen. Por tanto, futuras investigaciones deberían tratar de comprender la enorme diversidad de competencias en los jóvenes, si es que realmente queremos comprenderlos, así como estudiar por qué no tienen las que deseamos.

El siguiente desafío es de corte metodológico, a saber, la construcción de instrumentos que realmente tengan como objetivo determinar el desempeño de la gente joven en el mundo digital. Además, como plantean Calvani, Fini y Ranieri (2010), el constructo de las competencias digitales es tan amplio que seguramente no bastará con tan solo un instrumento.

En cuanto a las limitantes de este estudio, aunque he tratado de cubrir la mayor parte de los artículos relevantes, seguramente algunos habrán quedado por fuera. Por ejemplo, los artículos incluidos sólo cubrieron los escritos en inglés y español, pero es de esperar que en otros idiomas existan publicaciones relevantes. Lo mismo se puede decir de los estudios reportados en libros y literatura gris; esa información todavía está por analizarse. La otra gran limitante es producto del hecho de que el proceso de investigación haya sido realizado por un único investigador.

Finalmente digamos que 15 años de exploración del tema parecen muchos; no obstante, es tal su complejidad que hasta ahora tan solo estoy comenzando a cubrir su 
superficie. Esta agenda de investigación apenas comienza.

\section{Lista de referencias}

[Las referencias marcadas con un asterisco indican estudios incluidos en la metasíntesis (APA, 2010)]

Acosta-Silva, D. A. \& Muñoz, G. (2012). Juventud digital: Revisión de algunas aseveraciones negativas sobre la relación jóvenes-nuevas tecnologías. Revista Latinoamericana de Ciencias Sociales, Niñez y Juventud, 10 (1), pp. 107-130.

Acosta-Silva, D. A. \& Vasco, C. E. (2013). Habilidades, competencias y experticias: más allá del saber qué y del saber cómo. Bogotá, D. C.: Corporación Universitaria Unitec, Centro de Estudios Avanzados en Niñez y Juventud del Cinde y la Universidad de Manizales.

*Aesaert, K. \& van Braak, J. (2015). Gender and socioeconomic related differences in performance based ICT competences. Computers \& Education, 84, pp. 8-25. Doi:10.1016/j.compedu.2014.12.017.

*Arias, M., Torres, T. \& Yáñez, J. C. (2014). El desarrollo de competencias digitales en la educación superior. Historia y Comunicación Social, 19 (esp.), $p p$. 355-366. Doi:10.5209/rev HICS.2014. $v 19.44963$.

*Arras, A. M., Torres-Gastelú, C. A. \& GarcíaValcárcel, A. (2011). Competencias en tecnologías de información y comunicación (TIC) de los estudiantes universitarios. Revista Latina de Comunicación Social, (66), pp. 1-26. Doi:10.4185/RLCS-662011-927-130-152.

Barnett-Page, E., \& Thomas, J. (2009). Methods for the synthesis of qualitative research: a critical review. BMC medical research methodology, 9 (1). Doi:10.1186/14712288-9-59.

Bates, M. J. (1989). The design of browsing and berrypicking techniques for the online search interface. Online Review, 13 (5), pp. 407-424.

Bennett, S., Maton, K. \& Kervin, L. (2008). The «digital natives» debate: A critical review of the evidence. British Journal of Educational Technology, 39 (5), pp. $\quad$ 775-786. Doi:10.1111/j.14678535.2007.00793.x.

Bondas, T. \& Hall, E. O. (2007a). A decade of metasynthesis research in health sciences: A meta-method study. International Journal of Qualitative Studies on Health and Well-being, 2 (2), pp. 101-113. Doi:10.1080/17482620701251684.

Bondas, T. \& Hall, E. O. (2007b). Challenges in approaching metasynthesis research. Qualitative HealthResearch, 17(1), pp. 113121. Doi:10.1177/1049732306295879.

*Brazo, L., Ipiña, N. \& Zuberogoitia, A. (2011). Análisis de las competencias digitales de los estudiantes de tres títulos de grado de Mondragon Unibertsitatea. Edutec: Revista electrónica de tecnología educativa, (36), pp. 7-12.

Brunet, I. \& Belzunegui, Á. (2003). Flexibilidad y formación: una crítica sociológica al discurso de las competencias. Barcelona: Icaria.

Bullen, M., Morgan, T., Belfer, K. \& Qayyum, A. (2009). The net generation in higher education: Rhetoric and reality. International Journal of Excellence in ELearning, 2 (1), pp. 1-13.

Cabra-Torres, F. \& Marciales-Vivas, G. P. (2009). Nativos digitales: ¿ocultamiento de factores generadores de fracaso escolar? Revista Iberoamericana de Educación, (50), pp. 113-130.

*Calvani, A., Fini, A. \& Ranieri, M. (2010). Digital competence in $\mathrm{K}-12$. Theoretical models, assessment tools and empirical research. Anàlisi, (40), pp. 157-171. Recuperado de: http://ddd.uab.cat/ record/70680

*Calvani, A., Fini, A., Ranieri, M. \& Picci, P. (2012). Are young generations in secondary school digitally competent? A study on Italian teenagers. Computers \& Education, 58 (2), pp. 797-807. Doi:10.1016/j. compedu.2011.10.004.

*Carrasco, M. E. E., Sánchez, C. \& Carro, A. (2015). Las competencias digitales en estudiantes del posgrado en educación. 
Revista Lasallista de Investigación, 12 (2), pp. 10-18.

*Centeno, G. \& Cubo, S. (2013). Evaluación de la competencia digital y las actitudes hacia las TIC del alumnado universitario. Revista de Investigación Educativa, 31 (2), pp. 517-536. Doi:10.6018/rie.31.2.169271.

Comisión Europea (2007). Key competences for lifelong learning. European reference framework. Luxembourg: Office for Official Publications of the European Communities.

Dias da Silva, M., da Silva, D. \& do Amaral, S. L. (2014). The Y generation myth: Evidences based on the causality relations among age, diffusion and adoption of technology of college students of São Paulo State. Future Studies Research Journal: Trends and Strategies, 6 (1), pp. 32-52. Doi:10.7444/ fsrj.v6il.158.

Eshet-Alkali, Y. \& Amichai-Hamburger, Y. (2004). Experiments in Digital Literacy. CyberPsychology \& Behavior, 7 (4), pp. 421-429. Doi:10.1089/cpb.2004.7.421.

Esteve, F. M., Duch, J. \& Gisbert, M. (2014). Los aprendices digitales en la literatura científica: diseño y aplicación de una revisión sistemática entre 2001 y 2010. Pixel-Bit. Revista de Medios y Educación, (45), pp. 9-21.

Fajardo, I., Villalta, E. \& Salmerón, L. (2015). ¿Son realmente tan buenos los nativos digitales? Relación entre las habilidades digitales y la lectura digital. Anales de Psicología, 32 (1), p. 89. Doi: 10.6018/ analesps.32.1.185571.

Fan, X., Miller, B. C., Park, K. E., Winward, B. W., Christensen, M., Grotevant, H. D. \& Tai, R. H. (2006). An exploratory study about inaccuracy and invalidity in adolescent selfreport surveys. Field Methods, 18 (3), pp. 223-244. Doi:10.1177/152822X06289161.

Finlayson, K. W. \& Dixon, A. (2008). Qualitative meta-synthesis: a guide for the novice. Nurse researcher, 15 (2), pp. 59-71. Doi:10.7748/nr2008.01.15.2.59. c6330.

*García, J., García-Sánchez, J. N., ÁlvarezFernández, M. L. \& Díez-Caso, H. (2014). Efectos en la competencia digital tras la aplicación de un programa de competencias ocupacionales. European Journal of Education and Psychology, 7 (2), pp. 7381. Doi:10.1989/ejep.v7i2.180.

Gilster, P. (1997). Digital literacy. Nueva York: John Wiley.

Gui, M. \& Argentin, G. (2011). Digital skills of internet natives: Different forms of digital literacy in a random sample of northern Italian high school students. New Media \& Society, 13 (6), pp. 963-980. Doi:10.1177/1461444810389751.

*Guo, R. X., Dobson, T. \& Petrina, S. (2008). Digital natives, digital immigrants: An analysis of age and ICT competency in teacher education. Journal of Educational Computing Research, 38 (3), pp. 235-254. Doi:10.2190/EC.38.3.

Hargittai, E., \& Hinnant, A. (2008). Digital inequality differences in young adults' use of the Internet. Communication Research, 35 (5), pp. 602-621. Doi:10.1177/0093650208321782.

*Hatlevik, O. E., Guðmundsdóttir, G. B. \& Loi, M. (2015). Digital diversity among upper secondary students: A multilevel analysis of the relationship between cultural capital, self-efficacy, strategic use of information and digital competence. Computers \& Education, 81, 345-353. Doi:10.1016/j. compedu.2014.10.019.

Helsper,E.J.\& Eynon, R.(2010). Digital natives: where is the evidence? British Educational Research Journal, 36 (3), pp. 503-520. Doi:10.1080/01411920902989227.

Howe, N. \& Strauss, W. (2000). Millennials rising: The next great generation. Nueva York: Vintage Original.

Kennedy, G. E., Judd, T. S., Churchward, A., Gray, K. \& Krause, K.-L. (2008). First year students' experiences with technology: Are they really digital natives? Australasian Journal of Educational Technology, 24 (1), pp. 108-122.

*Li, Y. \& Ranieri, M. (2010). Are «digital natives» really digitally competent?: A study on Chinese teenagers: Chinese teenagers' digital competence. British Journal of Educational Technology, 41 
(6), pp. 1029-1042. Doi:10.1111/j.14678535.2009.01053.x.

Litt, E. (2013). Measuring users' internet skills: A review of past assessments and a look toward the future. New Media \& Society, 15 (4), pp. 612-630. Doi:10.1177/1461444813475424.

Livingstone, S., Bober, M. \& Helsper, E. J. (2005). Internet literacy among children and young people. Findings from the UK Children Go Online project. London: London School of Economics and Political Science.

Margaryan, A., Littlejohn, A. \& Vojt, G. (2011). Are digital natives a myth or reality? University students' use of digital technologies. Computers \& Education, 56 (2), pp. 429-440. Doi:10.1016/j. compedu.2010.09.004.

* Matamala, C. (2014). Factores predictivos de las competencias TIC en alumnos chilenos de secundaria. Revista Iberoamericana de Educación, 67 (1), pp. 121-136.

Miles, M., Huberman, M. \& Saldaña, J. (2014). Qualitative data analysis. Thousand Oaks: Sage.

Morduchowicz, R. (2008). La generación multimedia: significados, consumos $y$ prácticas culturales de los jóvenes. Buenos Aires: Paidós.

Oblinger, D. \& Oblinger, J. (2005). Educating the net generation. Washington, D. C.: Educause.

*Pino, M. \& Soto, J. (2010). Identificación del dominio de competencias digitales en el alumnado del grado de magisterio. Teoría de la Educación. Educación y Cultura en la Sociedad de la Información, 11 (3), pp. 336-362.

Prensky, M. (2001). Digital natives, digital immigrants part 1. The Horizon, 9 (5), pp. 1-6. Doi:10.1108/10748120110424816.

Ring, N., Ritchie, K., Mandava, L. \& Jepson, R. (2010). A guide to synthesising qualitative research for researchers undertaking health technology assessments and systematic reviews. Recuperado de: http:// www.nhshealthquality.org/nhsqis/8837. html
*Roig, R. \& Pascual, A. M. (2012). Las competencias digitales de los futuros docentes: un análisis con estudiantes de Magisterio de Educación Infantil de la Universidad de Alicante. @tic. revista d'innovació educativa, (9), pp. 53-60. Doi:10.7203/attic.9.1958.

*Romaniuk, M. W. (2015). Digital competences of Maria Grzegorzewska Academy of Special Education Students: Method and results of a survey. International Journal of Electronics and Telecommunications, 61 (3), pp. 267-272. Doi:10.1515/ eletel-2015-0035.

*Romero, M.\& Minelli, J. (2011). La generación net se tambalea: percepción del dominio de las TIC de estudiantes de magisterio. Teoría de la Educación. Educación y Cultura en la Sociedad de la Información, 12 (3), pp. 265-283.

Rychen, D. S. \& Salganik, L. H. (eds.) (2001). Defining and selecting key competencies. Seattle: Hogrefe \& Huber.

Sandelowski, M. \& Barroso, J. (2003). Classifyingthefindingsinqualitativestudies. Qualitative health research, 13 (7), pp.905923. Doi:10.1177/1049732303253488.

*San Nicolás, M. B., Fariña, E. \& Area, M. (2012). Competencias digitales del profesorado y alumnado en el desarrollo de la docencia virtual: el caso de la universidad de la laguna. Revista Historia de la Educación Latinoamericana, 14 (19), pp. 227-245. Doi:10.9757/Rhela.19.10.

Shaw, R. L., Booth, A., Sutton, A. J. Miller, T., Smith, J. A., Young, B. ... Dixon-Woods, M. (2004). Finding qualitative research: an evaluation of search strategies. $B M C$ Medical Research Methodology, 4 (5). Recuperado de: http://www.biomedcentral. com/1471-2288/4/5

Tapscott, D. (1998). Growing up digital: The rise of the net generation. Nueva York: McGraw-Hill.

Thinyane, H. (2010). Are digital natives a worldwide phenomenon? An investigation into South African first year students' use and experience with technology. Computers \& Education, 55 (3), pp. 1404-1404. Doi:10.1016/j.compedu2010.05.024. 
Timulak, L. (2009). Meta-analysis of qualitative studies: A tool for reviewing qualitative research findings in psychotherapy. Psychotherapy Research, 19 (4-5), pp. 591-600.

*Torres-Gastelú, C. A. (2015). Percepción de estudiantes universitarios sobre el modelo educativo y sus competencias en TIC. Educere, 19 (62), pp. 145-156.

*Torres-Gastelú, C. A., Domínguez, A. L., Flores, M. A., Kiss, G. \& Alejandre, A. R. (2015). Student's perception about online interaction, access and publishing content for academic use. Turkish Online Journal of Educational Technology, 14 (3), pp. 497-503.

Tupper, D. E. \& Cicerone, K. D. (1990). Introduction to the neuropsychology of everyday life. En D. E. Tupper \& K. D. Cicerone (eds.) The neuropsychology of everyday life: Assessment and basic competencies, (pp. 3-18). Boston: Kluwer Academic.

Van Deursen, A. J. A. M., van Dijk, J. A. G. M. \& Peters, O. (2011). Rethinking Internet skills: The contribution of gender, age, education, Internet experience, and hours online to medium-and content-related Internet skills. Poetics, 39 (2), pp. 125144. Doi:10.1016/j.poetic.2011.02.001.

Walsh, D. \& Downe, S. (2005). Meta-synthesis method for qualitative research: a literature review. Journal of advanced nursing, 50 (2), pp. 204-211. Doi:10.1111/j.13652648.2005.03380.x.

Zhao, S. (1991). Metatheory, metamethod, meta-data-analysis: What, why, and how? Sociological perspectives, 34 (3), pp. 377390. Doi:10.2307/1389517.

Zimic, S. (2009). Not so «techno-savvy»: Challenging the stereotypical images of the «Net generation». Digital Culture \& Education, 1 (2), pp. 129-144. 